\title{
Diálogo intercultural en Israel: elementos de la mediación transformativa
}

\author{
Jonatan LIPSKY \\ Graduado en la Universidad de Tel Aviv \\ lipskyj@gmail.com
}

Recibido: 04/06/2012

Aceptado: $13 / 11 / 2012$

\begin{abstract}
Resumen
La sociedad israelí, cuenta con un alto grado de segmentación social y una vida política frecuentemente controvertida, producto de su sociedad multicultural. Uno de las herramientas preponderantes para lidiar con el alto nivel de conflictos sociales, es la participación de jóvenes en grupos de encuentros que promueven el diálogo y la convivencia entre grupos sociales que se encuentran en conflicto.

Existen diversos enfoques y metodologías pedagógicas desarrolladas para los encuentros. Los encuentros entre árabes y judíos, que han sido desarrollados e investigados por décadas, nos permiten identificar los beneficios y perjuicios de los diversos modelos existentes. El presente artículo se refiere a ellos, para analizar y tipificar el modelo promovido por la Fundacion Guesher (Puente), la cual se ha focaliza en programas de diálogo religioso-laico, conflicto que ha llevado al asesinato en 1995, del entonces Primer Ministro Itzjak Rabin.

El presente estudio sugiere que dicho modelo se caracteriza por adoptar herramientas y técnicas de las variadas y diversas corrientes existentes en el ámbito del diálogo intercultural. Asimismo, se propone en el artículo, que la estructura del encuentro promovido por Guesher, su lógica de desarrollo y dinámica de trabajo, reflejan elementos característicos de la mediación transformativa.

El modelo aquí presente, se sostiene, genera una simpatía entre los participantes, a la vez que instan a una creciente responsabilidad civil. Por ello, se propone que este tipo de modelos sean empleados en sociedades de creciente polarización social, como es el caso de la sociedad israelí.
\end{abstract}

Palabras clave: dialogo intercultural, mediación transformativa, árabes, judíos, laicos religiosos, Israel

\section{Intercultural Dialogue in Israel: elements of transformative mediation}

\begin{abstract}
As a multiethnic, multicultural and multilingual society, Israeli society has a high degree of social segmentation. One of the main tools to deal with the prevailing high level of social conflict is fostering the participation of youth in encounter groups, which promote dialogue and coexistence between social groups in conflict.

Within the various approaches and teaching methods developed for encounters between Arabs and Jews, we can identify the pros and cons of each model. This article refers to them, analyzing and characterizing the model promoted by the Gesher Foundation (Bridge), which has fostered dialogue encounters on the secular-religious conflict that led to the murder of the Prime Minister Yitzhak Rabin in 1995.

The present study suggests that this model is characterized by adopting tools and techniques from the varied and various existing currents in the field of intercultural dialogue. It is also proposed in the
\end{abstract}


article that the structure of the meeting sponsored by Gesher, its logic and workflow development reflects characteristic elements of the transformative mediation.

This model, it is argued, creates a rapport between the participants while calling for civil responsibility. Therefore, this study suggests that such models should be employed in societies with increasing social polarization, as is the case with Israeli society.

Keywords: intercultural dialogue, transformative mediation, Arabs, Jews, secular, religious, Israel.

\section{Referencia normalizada}

Román Marugán, P. (2013). “La mediación política: concepto, procesos y problemáticas”. Política y Sociedad, Vol.50 núm. 1: páginas. 145-162

Sumario: Introducción. 1.Objetivos de los programas de diálogo entre árabes y judíos. 2.Reseña de los diversos modelos de diálogo desarrollados entre judíos y árabes. 3.Evaluando el proceso de diálogo. 4.Diálogo religioso - laico: el modelo de la Fundación Guesher. 5.Desarrollo del encuentro de Guesher. 6.Tipificando el modelo de Guesher. 7.Conclusión: mediación transformativa y el modelo de diálogo de Guesher. 8.Referencias bibliográficas

\section{Introducción}

Israel es el hogar de una población muy variada, con trasfondos étnicos, religiosos, culturales y sociales muy diversos, los cuales generan diariamente desafíos sociales. Las tensas relaciones entre la mayoría judía -cerca del 75\% de los más de 7 millones de ciudadanos- y la minoría árabe son ejemplo de ello. Asimismo, existen numerosos conflictos entre las distintas corrientes religiosas judías, llevando incluso en 1995 al asesinato del entonces Primer Ministro Itzjak Rabin, a manos de un colono judío.

Dado que en Israel el Estado es considerado ‘judío y democrático’ existen numerosos conflictos intergrupales producto de la definición del carácter del Estado. La vida pública israelí está reñida en el ámbito político y legal al aspecto religioso o nacional del concepto 'judío'. En este contexto, numerosos académicos han abordado la paradójica definición del Estado como judío y democrático. ${ }^{1}$

Como sociedad multiétnica, multicultural, multirreligiosa y multilingüe, Israel presenta un alto grado de segmentación social y una vida política frecuentemente controvertida. Existen numerosos intentos por mantener un nivel moderado de conflictos sociales, ya sea por medio del sistema judicial y político o a través de

${ }^{1}$ Por citar a algunos: Barak-Errez, D. (1998): A Jewish and democratic state, Tel Aviv: Tel Aviv University Press (Hebrew). Mautner, M. Sagi, A. \& Shamir, R. (1998): Multiculturalism in democratic and Jewish state, Tel-Aviv: Ramot. 
actividades realizadas por diferentes organismos que promueven el diálogo y la convivencia entre los diversos grupos sociales.

Se han llevado a cabo numerosos estudios e investigaciones con el fin de evaluar la eficacia de los distintos programas de diálogo entre árabes y judíos en Israel. Sin embargo, en el ámbito de los encuentros de diálogo laico-religioso no existe una tradición de investigación extensa.

El siguiente escrito tiene la intención de analizar en profundidad el modelo de los encuentros realizados por la Fundación Guesher (Puente), que ha promovido programas de diálogo religioso-laico durante los últimos 40 años. Yanay y LifshitzOron (2008: 288) sostienen que procesos como los realizados por la Fundación Guesher generan una simpatía entre los grupos, a la vez que instan a una creciente responsabilidad civil.

Para analizar en profundidad dicho modelo haremos uso de la literatura desarrollada en torno a los encuentros entre árabes y judíos. Se presentaran los diversos enfoques y metodologías pedagógicas desarrolladas para tales encuentros. El vasto conocimiento científico generado en aspectos de psicología social y en herramientas y técnicas del diálogo intergrupal, nos permite identificar los beneficios y perjuicios de cada modelo. Dicho conocimiento será utilizado para analizar el modelo de Guesher. El análisis de dichos encuentros se basa en materiales del archivo de la Fundación y en observaciones participativas realizadas durante numerosos encuentros.

A diferencia de los modelos de diálogo árabe-judío, no es posible tipificar el presente modelo en una escuela determinada ya que el mismo rescata una variedad de elementos y herramientas de diversas tradiciones.

Dichas características, y otras que serán analizadas a continuación, invitan a pensar en el modelo de Guesher en términos de la mediación transformativa. El presente estudio tiene como objetivo llenar el vacío existente entre estas dos tradiciones, la del diálogo entre grupos en conflicto y la mediación transformativa, proponiendo que modelos de diálogo de este tipo deben ser empleados en sociedades de creciente polarización social.

\section{Objetivos de los programas de diálogo entre árabes y judíos}

Con el fin de evaluar la eficacia de los distintos programas de diálogo entre árabes y judíos en Israel, se han llevado a cabo diversos estudios e investigaciones. Cada investigación, propone un concepto diverso del término 'diálogo', sobre el cual se evalúan los resultados de dichos encuentros. Por ello, previo a la evaluación, debemos preguntarnos ¿Cuáles son los objetivos de los encuentros? ¿Qué es considerado diálogo? ¿Cómo puede evaluarse el éxito o el fracaso en dichos procesos?

El concepto de diálogo establecido por Martin Buber y Carl Rogers, establece que el diálogo es más que un intercambio de información. Es un proceso de comunicación en el que los participantes no sólo comprenden "los procesos subjetivos del otro" sino que se comprende que el 'otro' forma parte del "mundo propio en el 
que uno vive”. El diálogo según Buber se basa en el reconocimiento que 'el otro es un ser único', 'que el otro está siendo sincero y expresando su realidad de un modo autentico' y 'el respeto por el otro debe nacer del principio que indica que el otro es una herramienta para nuestro propio desarrollo’ (Steinberg y Bar-On, 2002: 202).

Por su parte, teóricos constructivistas como Kenneth Gergen y sus colegas describen el diálogo entre grupos como un proceso de transformación crucial. El diálogo permite a los lados hacer frente a los desacuerdos o los conflictos planteados entre ellos a través de la auto-expresión y 'vivenciando' las emociones, experiencias, opiniones y valores del otro. Otro de los propósitos del proceso de diálogo es la reconstrucción de la identidad de los participantes, a través de herramientas y técnicas que caracterizan a los distintos modelos de diálogo.

\section{Reseña de los diversos modelos de diálogo desarrollados entre judíos y árabes}

A lo largo de la historia han existido diversos modelos de diálogo entre árabes y judíos en Israel. De hecho, se pueden identificar varias etapas en la evolución de los programas de convivencia y diálogo, producto de las circunstancias históricas y sociales del país, que reflejan las tendencias en las relaciones entre ambos grupos sociales. A continuación se presentan los diversos enfoques, herramientas y técnicas. Asimismo, se despliegan diversas perspectivas acerca de la evolución y desarrollo de los procesos grupales que acontecen en el marco de los grupos de diálogo.

Los diversos modelos existentes han sido clasificados y tipificados por sus objetivos y herramientas de trabajo. Según Katz y Kahanov se puede diferenciar entre los encuentros en el espíritu de las 'relaciones humanas', los encuentros de 'aprendizaje intercultural' y aquellos basados en un enfoque de 'resolución de conflictos'. Amir y Ben-Ari, proponen una clasificación similar de los encuentros según el modelo de 'contacto', el modelo de 'información', y el modelo 'psicodinámico' (Suleiman, 2004: 323).

La hipótesis del modelo de 'contacto' o modelo de 'relaciones humanas', en sus diversas formulaciones y elaboraciones, sugiere que se debe promover la interdependencia y la cooperación entre los participantes, garantizando la igualdad y eliminando la asimetría. Este modelo, desarrollado en gran medida por Allport (1954) y Pettigrew (1998) sugiere que a través de la interacción continua entre los participantes, se potencian la formación de amistades, lo cual puede ayudar a aliviar el conflicto entre los grupos y alentar el cambio en las actitudes negativas.

En Israel, decenas de organizaciones trabajan según este modelo en ámbitos como el arte, la música y el deporte, siendo Yehuda Amir, un referente de dichos programas. Las actividades, de interés general, atraen a decenas de miles de ciudadanos que trabajan juntos en espacios de interés común, en pos de objetivos compartidos.

Un modelo más sofisticado ha sido desarrollado en torno al aprendizaje del idioma. El lenguaje desempeña un papel crucial en la interacción social y la trans- 
misión de valores culturales y sociales, por ello el lenguaje puede ser considerado un recurso socio-cultural con el cual las naciones pueden unirse o separarse. Ejemplo de dicho modelo puede verse en el proyecto del Centro para la Educación Bilingüe en Israel, creado con el objetivo de fomentar la educación bilingüe, binacional y multicultural entre árabes y judíos.

A pesar de los éxitos que han tenido escuelas de este tipo, Zvi Bekerman y Gabriel Horenczyk (2004: 399) mencionan que han surgido numerosos problemas, producto de acontecimientos políticos que acontecen día a día. Así por ejemplo, presentan los investigadores presentan que en fechas como el Día de la Independencia de Israel o Día de la Catástrofe Palestina (Nakba), correspondientes al mismo evento histórico, las organizaciones no logran sobreponerse al desafío. Por ello, ceremonias de este tipo suelen realizarse por separado. Los autores destacan una declaración dada por los adultos involucrados en las escuelas bilingües, según la cual al final del día "nadie está realmente feliz".

Por su parte, el modelo de 'información' similar al modelo de 'aprendizaje intercultural', supone que el desarrollo de los estereotipos y prejuicios se debe a la insuficiente información o la desinformación sobre 'el otro'. Según este modelo, característico del período de la década de los '70, el encuentro ofrece una oportunidad para conocer y aclarar cuestiones culturales, costumbres e historia (Suleiman, 2004: 2005).

En aquella época, en un esfuerzo por explorar la posibilidad de integrarse en la vida diaria israelí, los líderes árabes alentaron la participación en las actividades de la convivencia. Esta etapa se caracterizó por un modelo que facilitó el "descubrimiento" de la cultura árabe, en ojos de los judíos. He aquí, que siendo los árabes minoría y conociendo ellos mejor la cultura judía, estos encuentros se centralizaron en la cultura árabe, dejando en claro que era preponderante el tono orientalista que adoptaban los encuentros a tal punto que fueron denominados encuentros de "humus y labane”, comidas tradicionales árabes (Abu Nimer, 2004: 409).

Durante la década de los ' 80 se desarrolló un nuevo modelo destinado a reducir los estereotipos culturales, priorizando la sensibilidad y comprensión. El modelo 'psicodinámico' suele caracterizarse por un enfoque en los aspectos del aquí y el ahora, y por ello se invita a los participantes a expresar libremente sus pensamientos y sentimientos, enfatizando las experiencias propias de participantes. En dichos encuentros los facilitadores tienden a remarcar las sensaciones comunes existentes en los dos grupos. Ramzi Suleiman (2004: 413) propone que es perjudicial la expectativa de un comportamiento similar por parte de los participantes judíos y árabes, evitando referirse a tratar eventos históricos.

Considerando esta crítica, hacia fines de los ' 80 , varias organizaciones comenzaron a emplear un enfoque pedagógico según el cual los participantes se enfrentaban a los temas en disputa, particularmente aquellos relacionados con actitudes e ideologías políticas. En la escuela Oasis de Paz (NSWAS) han desarrollado a lo largo de los años un modelo que trata de dar respuesta a este desafío, proporcionando herramientas de análisis (Abu-Nimer, 1999). 
Motivados por el trabajo de Helmes y Phinney, los encuentros patrocinados por esta escuela, proponen que el conflicto entre árabes y judíos es entre dos pueblos y dos identidades nacionales y no entre individuos. En este enfoque, los participantes se enfrentan a los temas en conflicto, particularmente aquellos relacionados con actitudes e ideologías políticas, como por ejemplo el modo en que se debe ser definido el Estado.

El objetivo del encuentro sólo se puede lograr a través de la clarificación de estas identidades. El encuentro ayuda a los árabes a ser conscientes que han interiorizado posiciones o conductas que reflejan el efecto de la opresión de la mayoría judía, proporcionándoles aquí una oportunidad para reconstruir su identidad. A su vez, los participantes judíos poseedores de un complejo de superioridad, creada por las relaciones estructurales inherentes, pueden enfrentarse a sus profundos temores y aprender acerca de las percepciones políticas y culturales de los árabes (Abu Nimer, 2004: 406).

Por último, el modelo de TRT (To Reflect and Trust), Reflejar y Confiar, desarrollado por Dan Bar-On, es conocido también como modelo de 'narración de historias'. El núcleo del proceso se puede definir como la construcción de un espacio seguro para los participantes, en el que pueden reflexionar juntos, con mutuo reconocimiento. Albeck, Adwan y Bar-On consideran que la falta de empatía es una barrera para la comprensión, por ello sostienen que el uso de historias personales es positivo ya que conducen a la comprensión del otro. El modelo propone que los participantes compartan sus experiencias personales, hablando desde el 'yo'. La historia personal (y familiar) permite, no solo conocerse personalmente, sino también facilita la representación de la historia colectiva, mejorando la capacidad de empatía hacia ‘el otro’ (2002: 318).

\section{Evaluando el proceso de diálogo}

Habiendo comprendido las principales estrategias, herramientas pedagógicas y objetivos de los diversos modelos, resta mencionar que los distintos enfoques generan diversas dinámicas grupales, lo que dificulta comprender el desarrollo de los seminarios de diálogo y los resultados de dichos encuentros.

Steinberg, Bar-On (2002: 201) rescatan dos teorías que describen los procesos que viven los grupos de diálogo. La primera teoría, desarrollada por Katz y Kahanov establece que el desarrollo se produce en cinco ejes en los que se produce una transformación, de individuo a grupo, de debate a diálogo, de dicotomía a complejidad, de cortesía a honradez y de dependencia al facilitador a la independencia. Los autores proponen que el progreso a lo largo de cada uno de estos ejes promueve un aumento en el entendimiento entre las partes (en Steinberg y Bar-On, 2002: 201).

El segundo modelo desarrollado por Halabi y Sonnenschein (2004), investiga el proceso ocurrido según el modelo de resolución de conflictos, aplicado en la Escuela Oasis de Paz. Los autores describen un modelo lineal. La primera etapa es identificada por los 'buenos modales', en la que se crea un ambiente de compañerismo y 
solidaridad frente a la difícil realidad exterior, a cuanta de honestidad y claridad de las identidades grupales. En la segunda etapa se produce la consolidación de los grupos nacionales y la lucha entre ellos. El grupo árabe comienza a ser más asertivo y exige centrarse en la conflictiva definición 'judía' del Estado. Frente a este importante desafío, el grupo judío busca focalizar el diálogo en los puntos débiles de la sociedad árabe, proponiendo una superioridad ética y moral, que permite 'restaurar el poder'.

En este punto el diálogo se encuentra en un 'callejón sin salida', siendo este momento el más agresivo del encuentro, caracterizado por un ambiente tenso de frustración en ambos lados. El punto muerto se rompe una vez que el grupo judío reconoce al grupo árabe, como parte del grupo nacional palestino, que ha sido oprimido. Dicho reconocimiento produce que el grupo árabe sienta menos necesidad de presentar un frente unido, dejando al descubierto las tácticas usadas y permitiendo que discurra de aquí en más el diálogo basado en el respeto mutuo.

Por su parte Steinberg y Bar-On (2002), disienten con el modelo lineal y proponen que el eje de desarrollo del grupo está ligado a la calidad del discurso. El discurso refleja cómo se percibe el cambio, tanto en el sujeto mismo, como en el otro.

Para ello los autores definieron seis categorías de calidad del discurso. En un extremo se encuentra el discurso 'etnocéntrico', en el que cada parte se concentra en sí mismo, estereotipando al otro. A continuación se encuentra la fase de 'ataque', en la que las declaraciones son percibidas como amenazas, pero a diferencia del discurso ‘etnocéntrico' habla hacia el otro y no solo de sí mismo. Luego la fase de ‘abrir una ventana', en la que se expresan los sentimientos y experiencias, sin ser capaces de comprender la realidad desde la perspectiva del otro.

A continuación existe la fase de 'reconocimiento de las diferencias' en la que se comprende que unos no entienden a los otros, generando un 'debate intelectual', conversación que puede conducir a la comprensión cognitiva del otro. Por último, se encuentra 'un momento dialógico', caracterizado por la reciprocidad, la igualdad y la escucha.

El vasto conocimiento adquirido en torno a los diversos enfoques, herramientas, técnicas y modelos de diálogo entre árabes y judíos en Israel, es producto de numerosas investigaciones realizadas a través de los años. Dicho conocimiento, que caracteriza y tipifica las prácticas existentes, será útil para analizar el modelo de diálogo propuesto por la Fundación Guesher, abocada al diálogo laico-religioso en Israel.

\section{Diálogo religioso - laico: el modelo de la Fundación Guesher}

El asesinato del Primer Ministro de Israel Itzjak Rabin en 1995, a manos de un extremista judío religioso, generó una profunda fractura entre laicos y religiosos en la sociedad israelí. Si bien durante décadas funcionaban instituciones y organizaciones cuyo propósito era tender un puente entre ambos grupos, desde dicha fecha, los programas de diálogo crecieron considerablemente. Si bien han habido numero- 
sos modelos de diálogo, a diferencia del caso de diálogo árabe-judío, las investigaciones realizadas al respecto en dicho ámbito son contadas.

Numerosos encuentros cercanos al modelo de 'resolución de conflictos', se realizan entre intelectuales y líderes religiosos y laicos con el propósito de convenir un nuevo contrato social que logre remplazar el sistema de acuerdos existentes en materia de religión y Estado. Ejemplo de ello, son los talleres liderados por personalidades como el Rabino Yaacov Medan y Ruth Gavison, fundadora y presidenta de la Asociación por los Derechos Civiles en Israel.

Por otra parte, un claro ejemplo del modelo de 'contacto', puede reconocerse en el programa de estudios que han impartido conjuntamente dos academias de estudios avanzados, una laica y uno religiosa, desde 1990. Tal como han presentado Sagee y sus colegas (2009) con respecto a este programa, la experiencia compartida genera cambios en la actitudes hacia 'el otro'. Asimismo, Gordon y Arian (2001: 203) han relevados datos de los participantes de los seminarios de diálogo promovidos por la Fundación Guesher, los cuales han revelado una profunda mejoría en la imagen percibida del 'otro'.

Sin embargo, tal como postula Ifat Maoz, académica israelí en el área de comunicación social, es muy limitado el conocimiento de los procesos que acontecen dentro del grupo, entre ellos los procesos relativos a relaciones de poder (2000: 150).

A continuación haremos un análisis del modelo de diálogo propuesto por la Fundación Guesher, de las dinámicas propuestas en sus talleres y objetivos, con el propósito de entender cómo es que se dan dichas transformaciones.

Durante los encuentros de Guehser participan adolescentes de 16 y 17 años. Los jóvenes religiosos estudian en Yeshivot y Midrashot (internados de estudio religioso para hombres y mujeres, respectivamente) identificadas con el movimiento religioso nacionalista, identificado con la población judía de colonos en Judea y Samaria. Por su parte, los jóvenes laicos, suelen pertenecer a colegios con notada orientación laica, tales como instituciones del movimiento kibutzeano, el cual es identificado con la izquierda política de Israel. En ambos casos, se trata de jóvenes provenientes de hogares de nivel socioeconómico mediano y alto, en donde los padres suelen ser profesionales con títulos académicos

Los encuentros suelen realizarse durante los días jueves, viernes y sábados, con el fin de experimentar juntos el espíritu sabático, de vasta importancia en la tradición judía. Los encuentros se realizan en un lugar neutro que permita a ambos grupos sentirse a gusto. Para obtener dicho objetivo, y a pedido de las instituciones religiosas, se dividen los encuentros de hombres y mujeres, en lugares diferenciados.

El encuentro se basa en dos ejes principales. Uno, analizar la identidad judía individual de los participantes, sus valores y creencias. Y segundo, analizar la identidad pública del país y los diversos modelos de convivencia posibles. Según los organizadores el objetivo es clarificar la identidad judía e israelí de cada participante, según su propia ideología. Asimismo objetivos principales de los encuentros son reducir los estereotipos, fortalecer el mutuo respeto y fomentar la cooperación y colaboración entre ambos grupos. 


\section{Desarrollo del encuentro de Guesher}

Los encuentros comienzan con una apertura compartida en la que se da la bienvenida a los aproximadamente 200 participantes, a los que se los dividen en grupos de no más de 20 participantes, procurando que el número de laicos y religiosos sea similar. Cada grupo es acompañado por un facilitador, religioso o laico, quien estará a cargo del grupo durante todo el seminario.

1) El primer taller que se realiza promueve la creación de un ambiente agradable y abierto. Para ello, el taller se inicia con dinámicas grupales que permiten que los miembros del grupo se conozcan unos a otro, a nivel personal. Durante este taller se estipulan las normas de comportamiento, se escuchan las expectativas de los participantes y se clarifica el rol que va a ocupar el facilitador. En esta instancia se resalta la importancia de la voz individual de todos y cada uno de los participantes, con el propósito de apoyar la variedad de opiniones y puntos de vista.

2) Seguido al taller de conocimiento interpersonal, se aborda la cuestión de los estereotipos y las ideas preconcebidas. La dinámica realizada invita a los dos grupos a presentar los estereotipos que existen hacia el otro grupo y aquellos que ellos creen que el otro tiene hacia el propio. Dos objetivos importantes existen en esta actividad, por un lado, comprender que el mecanismo psicológico que construye los estereotipos, es una herramienta indispensable que clasifica toda la información que el sujeto incorpora o asimila. Por el otro, el ejercicio permite especular qué piensa un sujeto laico de un religioso y viceversa. Así, los participantes comprenden que deberán corroborar consigo mismos en qué medida los estereotipos son correctos o no, a través de un diálogo sincero basado en el interés genuino en conocer al otro.

3) En el marco de esta primera etapa, caracterizada por la de falta de conocimiento de unos con otros, se realizan variadas actividades que promueven el trabajo en equipo, la coordinación y cooperación. Este tercer taller, propone que existen circunstancias en las que la cooperación es necesaria y positiva más allá de las diferencias.

4) Habiéndose establecido las pautas básicas de trabajo, se realiza un taller que tiene como propósito que los participantes examinen cuales son los valores que los guían en su vida. Siendo que el encuentro pondrá de manifiesto las diferencias en el tipo de vida que llevan los participantes, es de suma importancia reconocer el origen de dichas diferencias. El análisis de la escala de valores permite aclarar los diversos puntos de partida del que parten los participantes.

5) A posteriori a dicho ejercicio, se solicita que los participantes definan su identidad judía. Las diversas respuesta obtenidas, reflejan la brecha entre los participantes. Si bien se insta a respetar la diversidad de opiniones, a esta altura del proceso, no cabe duda, que los participantes juzgan y critican las opiniones ajenas. Este taller, refleja el paso a la segunda etapa en el desen- 
volvimiento del grupo, caracterizada por el distanciamiento entre los participantes, producto de sus creencias y puntos de vistas.

6) La jornada resulta pues, intensa. Los participantes realizan un resumen del día en grupos homogéneos, en los cuales los participantes religiosos se reúnen por un lado con un facilitador religioso, de igual forma que los participantes laicos. El objetivo de dicho taller es permitir que los participantes puedan compartir, hablar, canalizar sobre lo acaecido y así liberar el estrés generado durante el día. Asimismo se propone un trabajo de introspección dirigido a capitalizar lo experimentado por cada participante ya sea a nivel vivencial, emocional y conceptual. Considerando estos elementos, los participantes son invitados a realizar una reevaluación de sus objetivos, en vista del desarrollo de los dos próximos días del seminario.

7) El segundo día estará caracterizado por profundos debates, originados en las diversas percepciones de la religión. A partir de la parábola de La Caverna de Platón, se discute sobre los conceptos de 'verdad única', 'verdades múltiples' y 'verdades relativas'. Así, se comienza a comprender en qué medida los individuos, y los grupos, cuentan con una mirada maniquea o pluralista. De aquí, que aquellos que abogan por el concepto de una 'verdad única', no estarán dispuestos a corromperla. El séptimo taller, sirve para comprender en profundidad que no sólo se trata de diferencias de valores o costumbres, sino de perspectivas opuestas en relación a la concepción de lo que se debe o no.

8) Con el propósito de permitir un pequeño descanso a los participantes, a continuación se proyecta un capítulo de una serie televisiva israelí para adolescentes que resalta dichos dilemas y tópicos del conflicto de religión y Estado. Dichos tópicos de carácter público, son un ejemplo del desafío de la convivencia entre laicos y religiosos hoy en día en la sociedad israelí. En este caso, la participación pasiva que propone la proyección, invita en cierta forma a presenciar los punzantes dilemas desde un lugar curioso y simpático, como suelen ser transmitidos en las producciones televisivas.

9) Sin embargo, a continuación, durante el noveno taller, los participantes son invitados a debatir acerca de diversas situaciones que acechan a la sociedad israelí. ¿Deben permitirse o prohibirse los servicios públicos como museos y transporte público durante el día sábado, día observado por la religión como día de descanso en el que está prohibido realizar todo tipo de actividades? ¿ $Y$ los servicios privados? ¿Debería ser prohibida la cría, venta y consumo de cerdo en Israel, tal como propone la religión o debe prevalecer el derecho del individuo a consumir los que se le antoje? ¿Cómo debería procederse con un soldado religioso que se rehúsa por razones ideológicas a participar en el desmantelamiento de asentamientos de colonos judíos? ¿Cuál es la definición de ‘judío' que debe ser adoptada frente a las instituciones del Estado? ¿Acaso aquella que propone el Rabinato que designa como judío únicamente quien ha nacido de una madre judía o acaso debe definirse a partir del concepto de la ley de inmigraciones, según la cual judío es quien tenga al menos un abuelo o abuela judío, definición que data de las leyes de Nurenberg, utilizada en 
el período de persecución a los judíos por el por el nazismo. Dichos debates, son ejemplos de los dilemas existentes entre religiosos y laicos que conviven en el mismo país, ciudad, barrio o complejo habitacional. Durante este ejercicio cada participante pone a prueba sus 'líneas rojas’ y su capacidad de flexibilidad en aquellos dilemas del Estado ‘judío’ y 'democrático'.

10)Considerando que el debate en torno a dichas cuestiones es el punto máximo en el que se ven reflejado las diferencias entre los grupos, de aquí en más el seminario tiende a focalizarse en los puntos compartidos entre las partes. Durante el décimo taller, los participantes relatan en primera instancia cómo cada uno vivencia el día público de descanso, el sábado. Las concepciones son muy diversas según los grupos, esparcimiento y diversión caracteriza el relato de los laicos y de suma santidad para los religiosos. Este taller se realiza en vísperas del comienzo de día sábado, lo que obliga a los participantes a definir conjuntamente y en forma práctica cómo experimentarán conjunta-mente dicha jornada. El intento por respetar las costumbres y modalidades de ambos grupos, exige no solo debatir en torno a los dilemas sino, lidiar con ellos y poner a prueba la capacidad de vivir conjuntamente.

Considerando que el inicio del día sabático suele ser celebrado con ceremonias de carácter meramente religiosas, se invita a los participantes laicos a crear una ceremonia que refleje su percepción del día sábado. Esta ceremonia en la que participan ambos, religiosos y laicos, tiene como propósito experimentar un momento conjunto, el cual suele llamar a valores universales como el descanso del trabajo, la paz y el estar junto a los seres queridos. A continuación, se realiza la tradicional ceremonia religiosa, dando libertad a participar a quienes quieran, tal como sucede en cada uno de los tres rezos diarios que indica el judaísmo religioso.

Habiendo traspasado un mojón importante en el proceso de diálogo, al concluir la festiva y emotiva cena sabática, se invita a los participantes a dialogar en forma distendida y desestructurada. En esta oportunidad los participantes se agrupan de forma voluntaria, sin la interferencia de terceros, siendo los participantes quienes dirigen las conversaciones, preguntan y responden a sus pares. Estas conversaciones suelen extenderse hasta tardías horas de la noche. En ella los participantes, se focalizan en temas tales como 'destino divino', 'libre albedrío', 'sexualidad', 'homosexualidad', 'árabes en la sociedad israelí' o cualquier otro tema que los propios participantes proponen.

11)Durante el día sábado se realizan únicamente dos talleres, respetando así el día de descanso de los participantes y dando lugar a momentos de esparcimiento y espacios interpersonales. Por la mañana se lleva a cabo un taller de lectura de la porción correspondiente de la Biblia, ejercicio que tiene como propósito enfatizar el beneficio que existe tanto en la lectura crítica que caracteriza al lector laico, como de la lectura metódica del religioso, dando por sentado que el texto bíblico no es propiedad de unos o de otros y subrayando el común denominador que une a unos y otros. 
12)El último taller, décimo segundo en su número, que se realiza antes del cierre del seminario, se focaliza en problemáticas sociales de índole general que acontecen en la sociedad israelí: violencia, crimen, abusos y pobreza. El diálogo en torno a dichas temáticas, expresa la posibilidad de cooperación entre laicos y religiosos, basándose en el valor universal de solidaridad y justicia social. Dicho valor es sugerido como respuesta a los procesos de segregación y distanciamiento que caracterizan a la sociedad israelí.

Con la salida de la primera estrella del día sábado se realiza una ceremonia que, si bien cuenta con ciertos elementos religiosos, invita a una serie de cantos y festejos que simbolizan tanto la despedida y cierre del encuentro, como el comienzo de la semana (dado que en Israel el día domingo es laborable).

El desarrollo de los tres días de encuentro, tal como ha sido descrito aquí, requiere de un análisis que nos permita comprender la lógica que existe entre los diversos talleres. Por ello a continuación tipificaremos la experiencia del modelo de diálogo laico-religioso, a partir de los modelos de diálogo intergrupal, presentados anteriormente.

\section{Tipificando el modelo de Guesher}

En la apertura de este artículo se sostiene que el modelo de diálogo propuesto por Guesher no responde a una de las escuelas presentadas, sino más bien que rescata elementos de diversos modelos. A continuación se presenta una tipificación de los diversos talleres, según las herramientas y técnicas utilizadas a lo largo del encuentro y los objetivos que caracterizan a cada uno de los talleres.

Es posible identificar talleres cercanos al modelo de 'contacto' que promueve la interdependencia y la cooperación entre los participantes, tales como los talleres 3 , 11 y 12, donde los participantes trabajan en pos de un objetivo común, dejando de lado el conflicto latente entre ellos.

Asimismo se pueden identificar talleres cercanos al modelo de 'información', o al denominado modelo de 'aprendizaje intercultural'. En ambos casos, tal como se ha mencionado anteriormente por la teoría, se propone conocer al 'otro' para evitar estereotipos y prejuicios. Ejemplo de ello son los talleres 1, 2, 4, 5, 7 y 10 los cuales ponen en manifiesto dicha técnica, invitando a los participantes a conocer en profundidad el mundo del 'otro'.

El taller número 6, que sirve como momento de auto reflexión y análisis de lo acontecido hasta dicho momento, se asimila al modelo 'psicodinámico'. Aquí la propuesta es que los participantes expresen libremente sus pensamientos y sentimientos de los acontecimientos durante el aquí y el ahora. Dando lugar así a la sensibilización y la comprensión de sí mismos y del otro, desde un lugar que pone en manifiesto la identidad grupal de los participantes.

El modelo desarrollado por la Escuela Oasis de Paz, que se focaliza en el conflicto entre dos grupos y dos identidades, se ve reflejado en los talleres 7, 8 y 9. Tal 
como plantea el enfoque, aquí los participantes se enfrentan a los temas de conflicto, particularmente aquellos relacionados con actitudes e ideologías políticas, producto de las identidades colectivas que se han construido durante el encuentro.

Por su parte, el modelo de 'narración de historias' o TRT, en el que se hace uso de la historia personal para facilitar la representación de la historia colectiva, se ve reflejado en el taller numero 10 . Dicho taller, invita a comprender un tópico político e ideológico, desde una perspectiva personal, narrativa, que refleja una ideología del grupo de pertenencia.

Halabi y Sonnenschein (2004: 374) proponen tipificar los programas de diálogo según dos ejes principales. El primero tiene en un extremo los modelos de 'relaciones humanas', caracterizados por el énfasis en los aspectos psicológicos de la experiencia del encuentro en sí, mientras que en el otro extremo se encuentran los talleres de resolución de conflictos, que suelen focalizarse en la situación política, a la cual se requiere dar solución. El segundo eje, propone por un lado la deconstrucción de la dimensión del grupo en favor de contacto individual, mientras que el otro polo propone el fortalecimiento de la dimensión del grupo de pertenencia, como herramienta de trabajo.

A continuación se rescata la idea propuesta por los investigadores, pero en vez de tipificar el encuentro en sí, se propone tipificar los diversos talleres existentes durante el seminario de Guesher .

Gráfico 1: Tipificación de los talleres del modelo de diálogo de Guesher

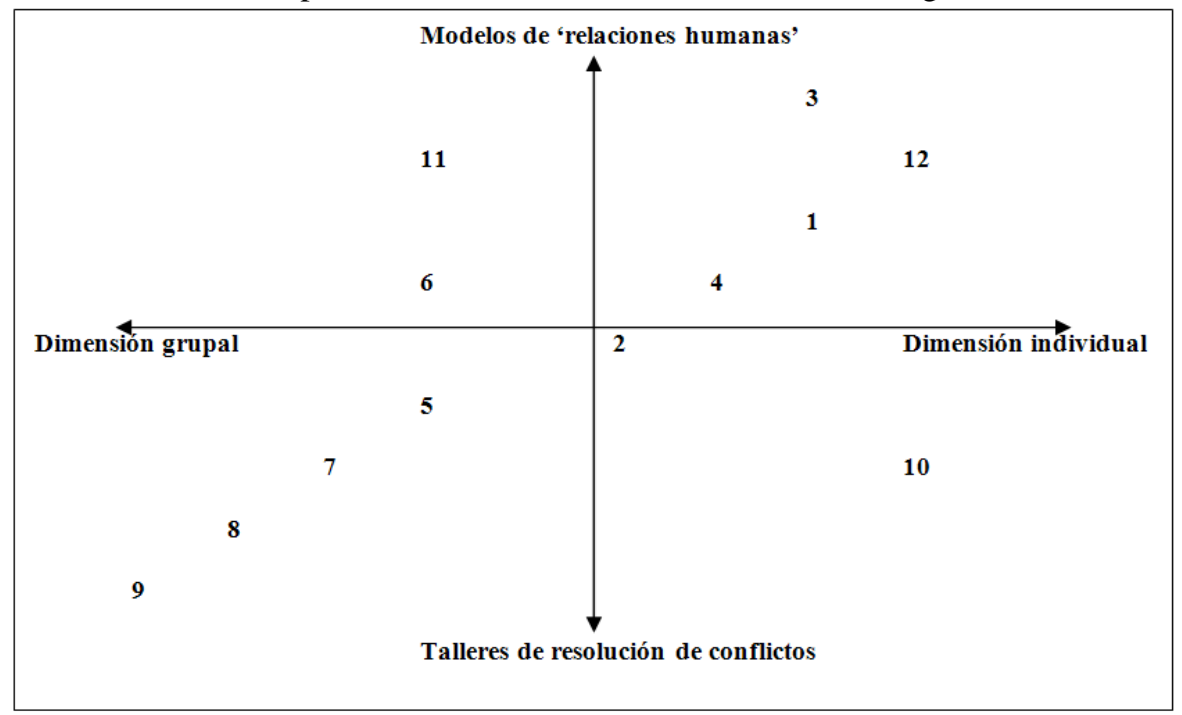

Comprendiendo la naturaleza de los distintos talleres se pueden identificar cuatro etapas en el desarrollo propuesto por este modelo de la Fundación Guesher. La primera etapa se caracteriza por la alineación y falta de conocimiento entre las 
partes, por lo que se puede entender la necesidad de emplear técnicas del 'modelo de relaciones humanas' en su dimensión individual. La segunda etapa, caracterizada por un paulatino conocimiento, comienza a poner de manifiesto la pertenencia grupal de los participantes, a medida que comienzan a debatir cuestiones de índole ideológicas.

En una tercera etapa se cristalizan las identidades grupales, producto de debates meramente políticos de orden público. La dinámica del debate llega al punto culmine en que los participantes deben adoptar herramientas del modelo de 'resolución de conflictos', el cual los insta a llegar a un acuerdo, relevante a las normas de convivencia durante el día sábado. A ésta instancia, en la cual los participantes deben encontrar una solución, la siguen los últimos tres talleres, que rescatan elementos del modelos de 'relaciones humanas', los cuales invitan a la cooperación y unión entre las partes.

Dicho desarrollo (representado en el gráfico número 2), podría recordar en cierta medida el modelo de la mediación clásica, según el cual el desarrollo estructurado del proceso permite avanzar hacia el objetivo de la resolución del conflicto. De acuerdo con Fisher y Ury (1981) el conflicto radica en la incompatibilidad de los intereses de ambas partes. A partir de la comprensión y el análisis de la causa u origen del conflicto, se promueve la colaboración, de la cual puede surgir un acuerdo y así resolver el conflicto.

Gráfico 2: Cuatro etapas en el desarrollo del modelo de diálogo de Guesher

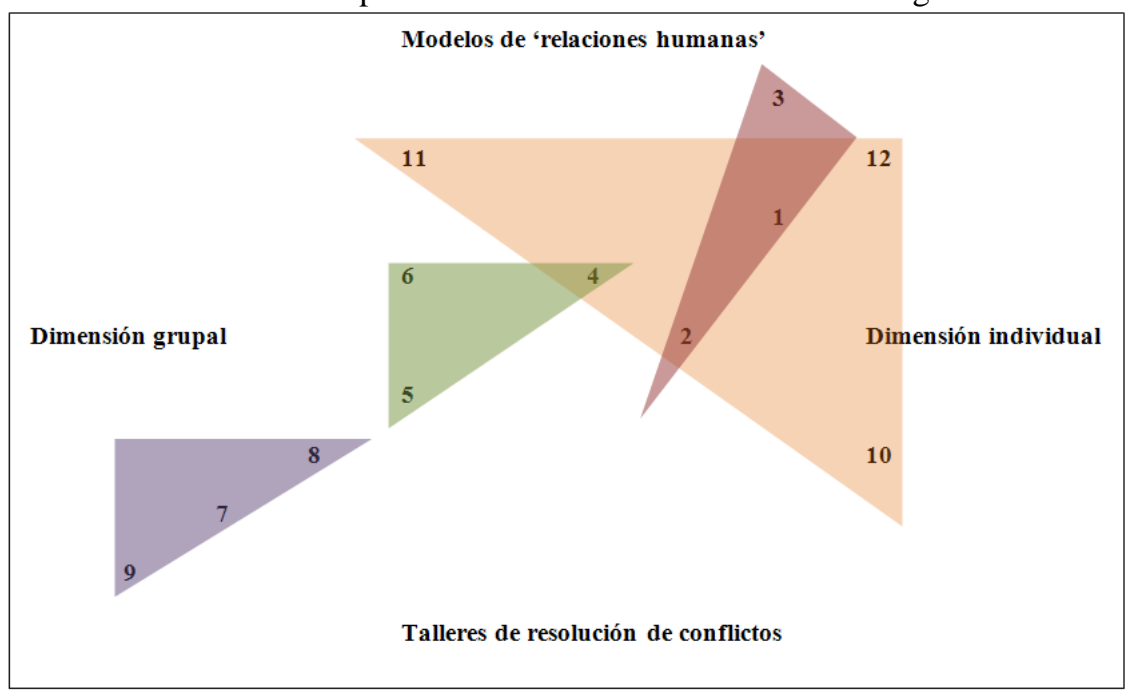

Sin embargo, debemos recordar que durante el proceso de diálogo entre laicos y religiosos no se generan acuerdo o soluciones concretas. Es más, la propuesta de cooperación en determinados ámbitos, de común interés, está establecida por la estructura misma del encuentro, predeterminada y planificada de antemano. Lo que 
sí es significativo en el proceso que propone el modelo de Guesher es la transformación de las relaciones entre los grupos. A continuación y a modo de conclusión se analizan las transformaciones que impulsan los encuentros de diálogo de Guesher, las cuales recuerdan en gran medida el modelo de mediación transformativa, desarrollado por Bush y Folger (1994).

\section{Conclusión: mediación transformativa y el modelo de diálogo de Guesher}

El modelo transformativo, desarrollado en la emblemática obra 'La promesa de la mediación', de Bush y Folger (1994) sostiene que el conflicto expresa un patrón de relación que mantienen las partes involucradas. Es decir, el conflicto está instalado y alimentado por la forma en la que se vinculan las partes. Esta estrategia no se centra en el establecimiento de un acuerdo determinado sobre un conflicto, sino más bien en la mejora o transformación de las relaciones humanas; el acuerdo no es un objetivo inmediato, sino que es la consecuencia de la transformación de las relaciones humanas.

El eje que guía a este modelo es cómo afrontar el conflicto a través del fortalecimiento (empowerment) propio y el reconocimiento a los 'otros'. El fortalecimiento debe darse en aspectos como las metas, alternativas, habilidades, recursos y procesos de decisión de uno mismo. Además, debe existir un reconocimiento activo hacia el otro, en forma verbal y en actitudes concretas. El modelo entiende y exige que durante el proceso las partes vivencien un proceso de crecimiento, asumiendo el protagonismo y control del mismo.

En este sentido, la calidad del discurso final refleja el proceso que han vivido los participantes, fortaleciendo sus propias posiciones y reconociendo a la vez la existencia de un 'otro'. La teoría propuesta por Steinberg y Bar-On (2002), que se remite a formas discursivas es útil aquí para identificar la transformación que viven los participantes.

En primera instancia se produce un discurso etnocéntrico, donde cada parte se concentra en sí mismo, estereotipando al otro y por ello, el segundo taller, pone énfasis en romper dicha forma discursiva. A continuación surge un discurso en el que se expresan los sentimientos y experiencias, sin ser capaces de comprender todavía la realidad desde la perspectiva del otro. Dicho discurso llamado por los autores 'abrir una ventana' es característico de los talleres 4 y 5 , donde se escucha al 'otro', ocurre en paralelo al discurso del 'reconocimiento de las diferencias', el cual refleja que no es posible reconocer y asimilar el discurso del ajeno.

La etapa de 'ataque' se puede percibir en los talleres 8 y 9, en paralelo a conversaciones que puede conducir a la comprensión cognitiva del otro, tal como proponen los talleres 7 y 10, dando lugar a un 'debate intelectual'. La posibilidad de mantener un 'momento dialógico', punto culmine de la calidad discursiva, puede darse en los últimos dos talleres. En este espacio se permite la reciprocidad, igualdad y escucha, considerando que en esta instancia los tópicos ideológicos ya han 
sido tratados. Así, se invita a cada grupo e individuo a ofrecer lo mejor de sí en ámbitos de cooperación.

Asimismo se puede reconocer una transformación en las cinco categorías propuestas por Katz y Kahanov (en Steinberg y Bar-On, 2002: 201). Mientras que al comenzar el encuentro se enfatiza el lugar de las creencias, valores e ideales del individuo, al concluir el encuentro se insta a la cooperación y colaboración colectiva, borrando la identidad individual o grupal. Sumado a ello, y tal como se presentó anteriormente, acontece una transformación del discurso, pasando de 'debate' al 'diálogo', dando lugar incluso a conversaciones de carácter íntimo o personal, caracterizadas por un intento de empatía y comprensión.

Otra transformación evidente se da en materia del paso de una perspectiva dicotómica a una perspectiva compleja. Esta, permite reconocer la existencia de un crisol de opiniones e identidades, refutando la imagen monolítica que existía previamente del 'otro'.

Asimismo, se puede identificar que de la actitud inicial de cortesía hacia el otro, reflejo de un respeto formal, por obligación, existe una transformación a un respeto real, identificado por la 'honradez', la cual está basada en valorar las concepciones ideológicas del 'otro', más allá de no compartirlas. Por último, una transformación vistosa se da con respecto a la dependencia de los participantes hacia el facilitador y al marco de diálogo propuesto por los talleres en sí.

Todas estas transformaciones, tal como proponen Katz y Kahanov, reflejan un significativo cambio en los participantes. El punto culminante de dicha transformación se ve reflejado quizás, luego de la cena sabática en la que se ven consumadas las más profundas y complejas conversaciones. La capacidad desarrollada por los participantes de dialogar en forma independiente, sin la intervención de terceros, que garanticen seguridad a los participantes, es el símbolo máximo del fortalecimiento propio y del reconocimiento hacía el otro.

Todo lo descripto anteriormente apunta a describir el proceso de transformación que pasan los participantes. El cambio es paulatino y escalonado, permitiendo avanzar en forma segura, pero no estrepitosa, hacia los puntos en conflicto. Luego de llevar el diálogo a lugares conflictivos, donde predominan cuestiones de identidad grupal o temas de índole ideológicos, se vuelve un paso hacia atrás, a los elementos de la identidad individual y herramientas de talleres de 'relaciones humanas'. Dicho vaivén tiene como objetivo evitar una fractura irreconciliable entre las partes, lo que permite finalizar el encuentro en un lugar de compromiso mutuo. 
Gráfico 3: Desarrollo creciente que permite fortalecimiento y reconocimiento, según el modelo de diálogo de Guesher

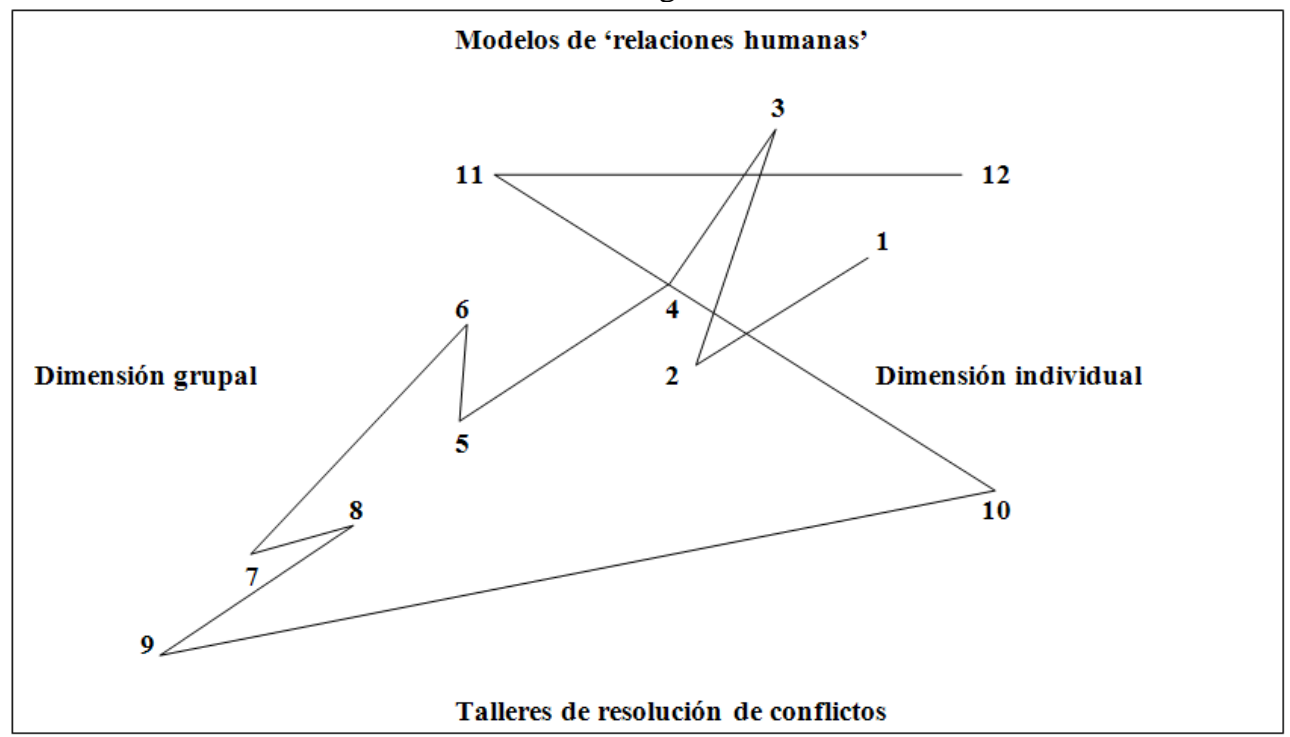

Tal como en la mediación transformativa hemos visto aquí, que en pos de conseguir el objetivo de mejorar la relación entre las partes deben emplearse variedad de técnicas, herramientas y estrategias. El desarrollo del proceso grupal no es lineal, sino que requiere de muchos vaivenes hasta alcanzar una transformación significativa.

Procesos como los realizados por la Fundación Guesher generan una simpatía entre los grupos, a la vez que instan a una creciente responsabilidad civil. Dichos procesos de diálogo construyen 'amistades', según las cuales todos somos 'otros', intentando prevenir la fragmentación de una sociedad por un lado y la homogenización por el otro.

\section{Referencias bibliográficas}

Abu-Nimer, M. (1999): Dialogue, conflict resolution and change: Arab-Jewish encounters in Israel, New York: State University of New York Press.

Abu-Nimer, M. (2004): 'Education for Coexistence and Arab-Jewish Encounters in Israel: Potential and Challenges', Journal of Social Issues, 60 (2): 405-422. 
Albeck, J. H., Adwan, S., \& Bar-On, D. (2002): 'Dialogue Groups: TRT's Guidelines for Working Through Intractable Conflicts by Personal Storytelling in Encounter Groups', Journal of Peace Psychology 8: 301-322.

Allport, G. W. (1954): The nature of prejudice, Wesley: Reading, MA.

Bush, R. A. B., \& Folger, J. P. (1994): The Promise of Mediation, San Francisco: Jossey-Bass.

Fisher, R., \& Ury, W. (1981): Getting to yes: Negotiating agreement without giving in, Boston, Mass.: Houghton Mifflin.

Gordon, C., \& Arian, A. (2001): 'Threat and Decision Making', Journal of Conflict Resolution 45 (2), 196-215.

Halabi, R. and Sonnenschein, N. (2004): 'The Jewish-Palestinian Encounter in a Time of Crisis', Journal of Social Issues 60 (2), 373-387.

Maoz, I. (2000): 'Multiple conflicts and competing agendas: A framework for conceptualizing structured encounters between groups in conflict-The case of a coexistence project of Jews and Palestinians in Israel', Journal of Peace Psychology 6, 135-156.

Maoz, I., Steinberg, S., Bar-On, D. \& Fakhereldeen, M. (2002): 'The dialogue between the 'Self' and the 'Other': A process analysis of Palestinian-Jewish encounters in Israel', Human Relations 55(8), 931-962.

Pettigrew, T. F. (1998): 'Intergroup contact theory', Annual Review of Psychology 49 (1), 65-85.

Sagee, R., Ezer, H., Shteiman, Y., Pniewsky, R. \& Tzabary, A. (2009): “"Peace begins with us. We are charged with educating an entire generation" - Graduates assess a joint religious-secular program at two colleges of education”, International Journal of Jewish Education Research 1 (3), 90-103 (en hebreo).

Steinberg, S. \& Bar-On, D. (2002): 'An analysis of the group process in encounters between Jews and Palestinians using a typology for discourse classification', International Journal of Intercultural Relations 26 (2), 199-214.

Suleiman, R. (2004): 'Planned Encounters Between Jewish and Palestinian Israelis: A Social-Psychological Perspective', Journal of Social Issues 60 (2), 323-337.

Yanay, N. \& Lifshitz-Oron, R. (2008): 'From consensual reconciliation to a discourse of friendship', Social Identities 14 (2), 275-292. 